\title{
Waste Management Using Machine Learning and Deep Learning Algorithms
}

\author{
Khan Nasik Sami, Zian Md Afique Amin, Raini Hassan \\ Department of Computer Science, Kulliyyah of ICT, International Islamic University Malaysia, Kuala Lumpur, Malaysia. \\ nasiksami@gmail.com, afiquezian@gmail.com, hrai@iium.edu.my
}

\begin{abstract}
Waste management is one of the essential issues that the world is currently facing, and it does not matter if the country is developed or underdeveloped. The key issue in this waste segregation is that the trash bin at open spots gets flooded well ahead of time before the beginning of the cleaning process. The cleaning process involves with the isolation of waste that could be due to unskilled workers, which is less effective, time-consuming, and not plausible because the reality is, there is a lot of waste. So, we are proposing an automated waste classification problem utilizing Machine Learning and Deep Learning algorithms. The goal of this task is to gather a dataset and arrange it into six classes consisting of glass, paper, metal, plastic, cardboard, and waste. The model that we have used are the classification models. For our research we did the comparisons between three Machine Learning algorithms, namely Support Vector Machine (SVM), Random Forest, and Decision Tree, and one Deep Learning algorithm called Convolutional Neural Network (CNN), to find the optimal algorithm that best fits for the waste classification solution. For our model, we found CNN accomplished high characterization on classification accuracy, which is around 90\%, while SVM indicated an excellent transformation to various kinds of waste, with $85 \%$ classification accuracy, and Random Forest and Decision Tree have accomplished 55\% and 65\% classification accuracy respectively.
\end{abstract}

Keywords - waste classification, image classification, machine learning, deep learning, random forest, support vector machine, decision tree, convolutional neural network, sustainable development goals.

\section{INTRODUCTION}

The production of waste has increased dramatically in recent times. If waste is not managed properly, it can have a calamitous effect on the environment. So, the sorting of waste should be done at the initial stage of waste management, to maximize the number of recyclable items and reduce the possibility of contamination by other items. The isolation of waste is done by unprofessional workers which is less effective, time-consuming, and not efficient because of a lot of waste. The world creates nearly one and half a billion tons of civil strong waste every year. As per the World Bank, and that figure is predicted to hit 2.2 billion tons by 2025 . Diversion of plastics from landfill to reusing can conceivably spare what might be compared to 60 million barrels of oil every year and lessen landfill volume necessities by up to $20 \%$. The U.S. Natural Protection Agency has suggested that source decrease, reusing, volume decrease, and landfilling be applied, in a specific order, in the treatment of city strong waste (MSW). Again, the economic value of waste is huge after it is segregated. The waste becomes valuable if it is segregated and recycled using the recent advancements in technology thereby becomes a useful entity. So, the execution of Artificial Intelligence and
Machine learning can carry a decent output for solving this alarming issue and to keep our environment a good place for all to live in.

In 2015, United Nations has created 17 Sustainable Development Goals (SDGs) which are also known as the global goals to end poverty, save the planet and ensure that all people enjoy peace and prosperity by 2030 . Based on the SDGs goals, we have taken our project as a milestone to solve some of the problems that almost all the nations are facing. In line with the United Nations Sustainable Development Goals, this project aids in realizing goal number 14 by wiping out the residues of plastic fishing nets, cigarette butts, and other categories of waste from the ocean coast [1]. Large marine mammals are washing up on shore dead, their bellies so full of plastic that they have starved. This research supports goal number 15 because life on land (SDG 15) can only be healthy when waste is properly managed. Again, waste is polluting the air when there is no management. With the burning of plastics everywhere the health impacts of open burning are catastrophic (SDG 3) [1]. Ensuring healthy lives and promoting well-being for all at all ages is the main aim of SDG3. Moreover, climate change, the impact of methane and $\mathrm{CO}_{2}$ from poorly managed waste will be a reason for up to a tenth of manmade greenhouse gas. 
So, it will hamper goal number 13, which indicates the climate impact (SDG 13) for our environment. And lastly, if we want clean water and proper sanitation (SDG 6), we need to be looking at waste to be managed properly [1]. So, our research carries a high value for sustainable development goals.

For conducting the study, a couple of research questions were formulated to guide the research, and they are as the following:

1) What are the waste categories for segregation in this research: Finding out whether a waste is paper, plastic, metal, glass materials or cardboard is the main target of this research. According to our training data provided in the dataset, we tried to determine the testing data be detected by comparing the attributes of given examples.

2) What are the key features determining the correct object: At first the model takes an input picture at that point and separate the locale for that. At that point, it compares the features with the past trained data, and toward the end, it orders whether the provided data or object is matched with the trained model and how much is the accuracy. To show signs of improvement accuracy it is smarter to do some increase in the preparation information, so the object can be analyzed with different angles and views. It is additionally better to do reshaping in the pre-processing stage to keep all cases in a similar size.

\section{Our research hypothesis is as the following:}

By implementing the classification algorithms: Support Vector Machine (SVM), Decision Tree, Random Forest and Convolutional Neural Network (CNN), the model helps to see how the items can fluctuate from one another relying upon different factors, for example, comparing the greyscale images or red-green-blue (RGB) value that can be ranged between 0 and 255 . These qualities would then be able to be placed into a cluster. Another way is separating the pictures into little pieces and afterward placing them into neural layers and every one of the neural systems is placed into an exhibit. At that point, we need to resize it while holding the subtleties of the picture. Then we must resize it while retaining the details of the image. After considering all the factors, the model predicts the accuracy of how likely an object match the trained sample.

Finally, the objectives of our research are given below:

1. To explore the dataset, which involves analyzing each feature variables to check if the variables are significant for building the model.

2. To visualize the dataset and identify the incorrect images.

3. To build the model that classifies the images and then sort them according to the classes
4. To do analyze the results based on performance evaluation

5. To find out the best suitable algorithm for this research problem

\section{RELATED WORKS}

Many different algorithms have been developed and used to solve the problem of classifying images properly. Some of them are CNN, SVM, Random Forest, Artificial Neural Network (ANN), Decision Tree, ResNet-50, VGG-16, AlexNet, $\mathrm{KNN}$, etc.

This section includes finding from the literature reviews that was done to get an idea of similar works on this domain. There are several pieces of literature that we referred to in this paper.

Along with the SVM algorithm, Random Forest Classifier, Gaussian Naive Bayes, and Multilayer Perceptron were used to detect different waste categories [2]. A diversified set of tree structures known as Bootstrap aggregating and selecting the right number of trees played a vital role in getting higher accuracy.

A different approach was taken with the use of IOT devices, ESP8266 Wi-Fi chip, Servo Motor, and Mobile app to automatically segregate the waste. Where IoT devices helped to count and monitor the type of waste and its quantity in mobile phones via an app [3].

Similarly, the study [4] shows the use of Raspberry Pi 3 B+, Raspberry Pi Camera v2, Servo Motor, Linkit One, IR Collision Sensor, Ultrasonic Sensor, Temperature and Humidity Sensor, GPS, AWS lo Core. All of them are connected and trained with a Machine learning model called Inception $\mathrm{V}_{3}$.

Another study [5] shows the use of Raspberry Pi, I293d Motor Driver, Webcam, DC motors, Inductive Sensors along with Machine Learning algorithm SVM solves the problem of segregating waste in domestic life.

The study by [6] introduces Computer Vision implementation along with Deep Learning. It used HOG, Gabor, Sliding window CNN, GarbNet (FCN with LRN). GarbNet with LRN processes six times faster output with better accuracy in this study. The model was deployed in a mobile app named SpotGarbage.

Different sizes of decision trees and random forest were applied in this experiment. SVM, Decision Tree, Random Forest were then again added with Principal Component Analysis (PCA) on the trashnet dataset.MobileNetV2 's lightweight neural network was trained by migration and transformation and used for extracting features, and the extracted features were integrated for classification into SVM [7]. This method solves the overfitting problem by implementing transfer learning.

Pearson Correlation Coefficient (PCC) and Spearman Rank Order Correlation Coefficient (SCC) were used to 
estimating the performance of neural networks. Among AlexNet, VGG-16, SVM, KNN, and Random Forest, VGG-16 has been the most accurate and computationally efficient model in the study [8].

Integrated sensor, microcontroller and IOT cellular module, Mobile app combined with J48, Random Tree, Random Forest classifier, K-means algorithms, and Ant Colony Optimization were implemented in the study [9].

After the updating of weights and bias by using Stochastic Gradient Descent with Momentum(SGDM) in the neural network, the fully connected layers were replaced by SVM [10]. This hybrid model was able to get high accuracy in identifying waste objects.

However, in [11], the researchers used a smaller filter quantity and size of AlexNet with Adam gradient descent optimization and the SVM for comparison.

Again, two popular learning algorithms were used which are CNN and SVM. The researchers chose $256 * 256$ colored png images and implement them on raspberry pi 3 [12]. It only compares two algorithms then finds out the best model with the measurement of speed and classifications.

For the classification system, several kinds of decision trees have been introduced to find active objects by multiwavelength data, such as REPTree, Random Tree, Decision Stump, Random Forest, J48, NBTree, AdTree [13]. These decision tree approaches are in the WEKA package. In the process of classification by decision tree methods, the classification rules are easily obtained, moreover, these methods are clear and easy to visualize.

Various random forest approaches also have been used in the field of image classifications. E.g., shape and appearances representations that support spatial pyramids, automatic selection of the regions of interest in training, and the use of random forests as a multi-way classifier [14].

The numerous low-level characteristic features such as color, shape, texture of the image form the various domains of the decision tree among others. The researchers discussed the algorithm to calculate the relative distance between the retrieved results as a subprocess required in this approach [15].

Another research has shown that the Python index package of Spyder is used to detect and segregate the waste material in real-time through a webcam [16]. The research proposes an intelligent waste classification system using the CNN algorithm, a Deep Learning based image classification model is used in the paper so that the bio and nonbiodegradable objects can be classified based on the object recognition accuracy in real-time. Open-source library tensor flow has been used in the research.

Multilayer hybrid deep-learning system (MHS) is another approach that can automatically sort waste of by individuals. This system deploys a high-resolution camera to capture images of waste and sensors to detect other useful details about the feature [17]. The MHS uses a CNN-based algorithm to extract image features and a method of consolidating image features and other feature details with multilayer perceptron (MLP) to identify waste as recyclable or other waste

\section{Methodology}

\section{A. Data Collection and Splits}

For the dataset, we have utilized the waste image dataset which was created by Gary Thung and Mindy Yang. Dataset was available in the internet sources and the owner of the dataset was permitted to use for any kind of research purpose. The dataset contains 2500 pictures of different garbage which are cardboards, metal, plastic, paper, glass, metals. We have divided the dataset into training, testing, and validation pictures. The training set comprises $80 \%$ and the test set contained $20 \%$ of the data.

\section{B. Tools}

We mainly used Google colab and python for our experimental setup and analysis process.

- Google Colab: Google Colab is a free cloud administration where we can do the coding part. One of the major features of the colab is that we can easily change the runtime. For our research as the dataset is big, we use GPU runtime. One can improve your Python programming language coding aptitudes.

- Python: Python is a programming language that we mainly used in this research. It is a high-level, objectoriented programming language with dynamic semantics. It has a syntax that allows developers to write programs in fewer lines comparing with other kinds of programming language. It has a verse utility in the field of GUI development, web development, scientific development, and software and system administration.

- Machine Learning algorithm(s) utilized, along with numerical equation(s).

\section{Algorithms}

To build our model on the trash image dataset, we have used four algorithms, which are the SVM, Random Forest, Decision Tree and CNN. The functionality of the classification algorithms is basic. You foresee the objective class by dissecting the preparation dataset. The following describe the technical details of each algorithm:

\section{- SVM:}

To solve binary classification problem, the SVM was developed [18]. The objective was to find the optimal hyperplane $f(w, x)=w . x+b$ to segregate two classes from the provided data, with the features $x \in \mathbb{R}^{m}$. By solving the optimization problem stated in (EQ. 1), SVM learns the parameter w. 


$$
\min \frac{1}{p} w^{T} w+C \sum_{i=1}^{p} \max \left(0,1-y_{i}^{\prime}\left(w^{T} x_{i}+b\right)\right)
$$

Here $w^{T} w$ w is the Manhattan norm(L1 norm),the penalty parameter is $C, y_{i}^{\prime}$ is the actual label, and $w^{T} x_{i}+b$ is the predictor function. The above equation is known as L1-SVM, with the standard hinge loss. More stable result is shown by the counterpart of L1-SVM, which is known as L2-SVM(Eq.2).

$$
\min \frac{1}{p}\|w\|_{2}^{2}+C \sum_{i=1}^{p} \max \left(0,1-y_{i}^{\prime}\left(w^{T} x_{i}+b\right)\right)^{2}
$$

Here $\|w\|_{2}^{2}$ refers to the Euclidean norm (L2 Norm), with the squared hinge loss.

\section{- Random Forest:}

As our problem was regarding to the classification problem, we used Gini index formula to decide how the nodes on a decision tree branch[10].

$$
\text { Gini }=1-\sum_{i=1}^{c}\left(P_{i}\right)^{2}
$$

To evaluate the Gini of each branch on a node, this formula uses the class and probability to determine which of the branches is more likely to occur. Here, $P_{i}$ is the relative frequency of the class in the dataset that we are studying, and $\mathrm{c}$ is the number of classes.

We have also used the entropy to decide how nodes branch in a decision tree.

$$
\text { Entropy }=\sum_{i=1}^{c}-P_{i} * \log _{2}\left(P_{i}\right)
$$

To decide about how the node should branch, Entropy uses the likelihood of a certain result. Owing to the logarithmic function used in calculating it, it is more mathematically intensive, unlike the Gini index.

\section{- Decision Tree:}

In Decision Tree, Information Gain is one of the Attribute to further describe the tree. It mitigates the data necessary to classify the data points into the respective partitions and represents the least randomness or "impurity" in those partitions.

$$
\begin{aligned}
& \operatorname{Info}(\mathrm{D})=-\sum_{0}^{m} p_{\mathrm{i}} \log _{2}\left(\mathrm{p}_{\mathrm{i}}\right) \\
& \operatorname{Info}_{A}(\mathrm{D})=\sum_{j=1}^{v} \frac{D j}{D} x \operatorname{Info}\left(\mathrm{D}_{\mathrm{i}}\right)
\end{aligned}
$$

Here [19], $\mathrm{p}_{\mathrm{i}}$ is the probability in the dataset $\mathrm{D}$ belongs to Class $\mathrm{Ci}$ and is estimated by $|\mathrm{Ci}, \mathrm{D}| /|\mathrm{D}|$. Info (D) is simply meaning the amount of information needed to identify the class of a Data point D. Info(D) is also known as the entropy of the dataset $D$.

The information gain can be calculated as follows:

$$
\operatorname{Gain}(A)=\operatorname{lnfo}(D)=\operatorname{lnfo}(D)
$$

- $\quad$ CNN:

CNN is individual in its 3D volumes of a neuron: width, height, and depth. CNN consists of a sequence of convolutional layer, pooling layers, fully connected layers. Only a small region of the previous layer will connect the neurons in the convolutional layer. The activation neurons of the layer in fully connected layers are fully connected to all activation neurons of the previous layer [13]. The fully connected function can be expressed as the following forward:

$$
\begin{aligned}
\sum X_{i}{ }^{L+1} & =\sum W_{j, i} i^{++1} X_{i}{ }^{L}, \\
g_{i}{ }^{L} & =\sum W_{j, i}{ }^{L+1} g_{j}{ }^{L}
\end{aligned}
$$

Where $\mathrm{X}_{\mathrm{i}}^{\mathrm{L}}$ and $\mathrm{g}_{\mathrm{i}}^{\mathrm{L}}$ indicates the activation and the gradient of neurons $i$ at layer $L$ and $W_{j, i} i^{L+1}$ is the weight connecting neurons $i$ at layer $L$ to neurons $j$ at layer $L+1$.

\section{Performance Measurement}

To measure the performance of our algorithms, we took help from some of the performance measures features such as confusion matrix, precision, recall, F1-score, accuracy, and cross-validation. We have defined them briefly next in the implementation part.

\section{E. Data Preparation \& Pre-Processing}

As the information utilized in this test is an assortment of waste-related pictures, there should be some preprocessing on them to change over the information in the configuration that can be taken care of to the Al models. Pictures in the training dataset had contrasting sizes, hence pictures must be resized before being utilized as a contribution to the model. Waste images were resized to the shape of $512 * 384$ pixels from the original dataset and moved to separate directory. We then converted our dataset into greyscale images with dimension of $50 * 50$ pixels increasing the computation speed. On this converted data, we have built our models.

\section{MODELLING}

\section{A. SVM}

SVM makes all the difference because it includes complex kernel groups such as RBF (Radial Bias Function), Neural Network class, and polynomial classifiers, yet it is easier to analyze SVM.

For the SVM in a high dimensional element that is nonlinearly connected to input space, it relates to a direct method, but in that high dimensional space, it does not involve any complex calculations. With the support of kernels, any single significant figure and calculation take place in the input space.

The minimum function is the Optimal Hyperplane indicator, which is accurately the maximum range of the separation distance between the two classes. By applying a quadratic function that makes it sufficiently streamlined to obtain the maximum optimized patterns lying on its margin, this hyperplane is formed. These examples are referred to as support vectors containing data related to classification.

As a result of its bits accessible for various types of information, SVM are advantageous for using it. With its default use, based on a hyperplane, it isolates two explicitly divisible groups. The LSVM (Linear SVM) is this kind of SVM. 
By considering the limits of a dataset, all the accessible preparing vectors are then split into a couple of groups and the hyperplane is chosen with the aim that these support vectors are at the base of the hyperplane. Therefore, according to SVMs, to classify any class rather than full training examples, only these support vectors are important.

As indicated by SVM, it is only these support vectors that are required to group any class instead of the total preparation models. $D+$ and $D$ usually mean the distinction between the support vectors and the hyperplanes, while the margin of the isolating hyperplane is the whole of each of these separations.

In this case, the assumption of linear separation of the data was not there because it was a multi-class classification problem. We may use a feature in such circumstances to change our information into a higher dimensional space. A simple polynomial function can be activated to access information to turn it into a parabola of information focuses. But this method can be very costly to pursue computationally and can therefore be used in such cases with a kernel trick. This associate with using a capacity that takes as its data the vectors in the first space and results in a spot outcome of the vectors in the component space. This converts the vectors from a nonlinear space to a linear space in the long term.

\section{B. Random Forest}

Random Forest is a very well-known classifier used for multi-class classification. It uses several number of distinct decision trees, and these trees work for randomization. The leaf nodes of each tree are the lowest level of the tree and are the distribution over the class of images. As it is sent down at each node and tree, the image is labeled, and the collected value is determined towards the end of leaf circulation.

In two different ways, randomization is a part of the calculation. One is by sub-sampling the dataset in the training period and choosing node tests. Inspecting methodology assumes a significant job in the outcome classification.

One of the study presented a contextual overview of three angles within the preparation test, which were test size, spatial autocorrelation, and class extent[20]. Random Forest Image Classification has demonstrated sensitivity to variables such as class proportions, sample size, and training data characteristics.

To improve execution and accuracy, Random Forest classifications should be reproduced in any event, it is already an ensemble approach to regression modelling and classification. Every algorithm has its benefits and drawbacks.

Random Forest advantage points include:
- $\quad$ SVM and Boosting calculations can be contrasted with easy-to-use parameters and are less sensitive to those parameters.

- Compared to individual decision trees, a smaller issue of overfitting and thus pruning of trees can be avoided.

- The accuracy is improved by the automated identification of outliers and essential variables and Random Forest is therefore much easier to use. Each advantage, however, also comes with its own set of limitations. Random Forest confinement has been investigated up until now is that prediction is limited to a specific range of response values in the test set due to regression trees. Thus, it turns out to be true that preparation information requires a full range of reaction factors and all examples ought to have all scope of reaction information esteems.

\section{Decision Tree}

The decision to make strategic divisions greatly influences the accuracy of a tree. For characterization and regression trees, the decision models are diverse. To choose to split a node into at least two sub-nodes, decision trees use various calculations. The homogeneity of the resulting sub-nodes is built by the formation of sub-nodes. As such, we can assume that the value of the nodes increases as far as the objective variable is concerned. From each available variable, it forms Decision Trees.

Decision Trees are divided into two groups, based on the target variables.

- Decision Trees Categorical Variable: This is where the algorithm has a goal categorical variable. In the process of classification, the decision tree learns from various features of the data, and it ends up at a leaf node of one of the six categories of targets after passing each data point through each node.

- Continuous Variable Decision Trees: In this case, the input features of the decision trees are used to estimate a continuous output. This is used to solve regression problems. splits the nodes and then selects the split that causes the most homogeneous sub-nodes.

\section{CNN}

CNN is one of the main parts of Neural Networks. It is used widely in image recognition and classification to detect various objects, recognize faces, and so on. CNN is made up of neurons that can learn weight and biases.

The components in CNN can be divided into few segments:

- Convolutional Layer: It is the first layer of CNN. Convolution is the primary layer to separate highlights from an input picture. Convolution 
protects the connection between pixels by learning images features utilizing little squares of information. It is a numerical activity that takes two sources of info, for example, an image matrix and a filter or kernel.

- Max Pooling Layer: Max pooling is a pooling activity that chooses the greatest element from the locale of the feature map covered by the channel. Thus, the output after the max-pooling layer would be an element map containing the most conspicuous highlights of the past component map. This layer manages to let the examining and preparing time.

- Dropout Layer: Dropout is a procedure used to keep a model from overfitting. Dropout works by randomly setting the active edges of hidden units (neurons that makeup concealed layers) to 0 at each update of the training stage. Normally, dropout is put on the completely associated layers simply because they are the ones with the more prominent number of parameters and in this manner, they are probably going to unnecessarily co-adjusting causing overfitting.

- $\quad$ Flattening Layer: After finishing the last stages, we should have a pooled include map at this point. As the name of this progression suggests, we are in a real sense going to flatten our pooled include map into a column. The explanation we do this is that we need to embed this information into an ANN later. What occurs after the leveling step is that you end up with a having long vector of input features that go through the ANN to have it prepared further.

- Dense Layer: Dense layers add an intriguing nonlinearity property, in this manner they can show any numerical function. Nonetheless, they are yet limited as in for a similar input vector we get consistently a similar output vector. They cannot identify reiteration as expected or produce various answers on a similar input.

- Fully Connected Layer: A fully connected layer mainly takes all the output from the previous pooling or convolution layer and depict the correct image or classify the correct label. The fully connected layer (flatten) receives the output from the previous layers and then flatten them and create a single vector that can be an input for the following stage. After receiving the input, the layer applies weight to anticipate the correct label. The fully connected output layer shows the last probability for each object.

\section{IMPLEMENTATION}

\section{A. SVM}

We have imported all the necessary libraries and specified the data categories. In the next step, we have joined all the subcategories of the data and resized and converted them to the vector type using the flatten function. Then we have saved the preprocessed data in pickle format so that we can use the saved pickle afterward. We have shuffled the data which is an important part of getting an unbiased result for the prediction.

After splitting the data into training and testing data, we created the model for SVM using SVC. For the first testing, we used the kernel to 'rbf', $C=10$, and kept the gamma to auto. Then for this configuration, the model was able to predict the waste with an accuracy of $86 \%$. Then we tried to optimize the model with hyperparameter tuning and for this, we have used the Grid Search method. It exhaustively generates candidates from a grid of parameter values. It goes to all the possible values provided. It took 22.4 minutes for our provided configurations to execute with 80 different possible outcome tests. Then they provided an accuracy of $86.2 \%$ which is the same as before. Then we tried again with it with 3 different kernel types, 4 different range of values for $C$. This time the accuracy was $85 \%$ but the score for the confusion matrix was better than before.

\section{B. Random Forest}

A random forest classifier is a model combining many decision trees. It samples random training data points while building the trees. It adds additional randomness in the model if the number of trees grows. It creates a forest with many trees that are not correlated. Random Forest is a bagging algorithm for achieving low-prediction error.

Firstly, we imported the random forest classifier after all the preprocessing with n_estimatiors $=10$ which means with ten trees, criterion as entropy, and with 50 random states. With this configuration, when we increased the number of estimators, the accuracy slightly increased. Then we tried to optimize it with a random search optimizer as the hyperparameter tuning technique. It takes random configurations from the provided options. It can narrow down the possible outcomes which reduce the computation time. For the cross-validation, we used a stratified K-fold validation technique where the randomness of the data is confirmed. It gave us the optimal configuration for testing.

The cross-validation score increased to 70 which was previously 65 . But the accuracy was unchanged which is $55 \%$ for our data set. Also, the other performance measurement scores were increased after tuning with random search.

\section{Decision Tree}

It provides the result as a graphical representation like a flowchart where each node of the tree represents test cases. Each branch represents the outcomes from the test and the nodes represent the class labels.

After all the preprocessing, we have made the classifier for the decision tree model. We used entropy as the criterion and the depth of the tree we gave the value as 10. Other 
parameters were chosen as the default ones. For this configuration, our model was able to predict the waste materials with $65 \%$ accuracy.

D. CNN

For CNN implementation, we first extracted our images from the zip file and then divided the images into classes. Then we have split the images into the train, test, and validation set. After that, we have visualized the dataset and create our CNN model. We have used Resnet34. It is known as a residual neural network which has a lot of layer inside it. It is already pre-trained in ImageNet Database. A pre-trained CNN performs better on new image datasets. Then we have selected the best parameter for our model using learn.Ir_find and it gave us the perfect learning rate to reduce the error rate. With the learning rate, we then trained our model. After training, we have visualized the mostly incorrect images that my model was not able to perform well. It is because the photos received too much exposure or something, and this is not the fault of the model.

Then we have used the confusion matrix to find our accuracy of the model, and we have found that our model is sometimes confused between glass and plastic, glass, and metal. Then we have predicted our model based on the actual images and observe that our model can successfully predict based on the actual images. We have ended up achieving an accuracy of $90 \%$.

\section{RESULt ANALYSIS}

\section{A. Accuracy}

Accuracy is the most important part of the measurement feature. As we have done the confusion matrix in our algorithms. That is why our accuracy measurement has been done by a confusion matrix. The accuracy comparison found in our experiment is shown in Fig.1, where it shows that CNN and SVM achieved an accuracy of $90 \%$ and $85 \%$ respectively where random forest and decision tree shows only $55 \%$ and $65 \%$.

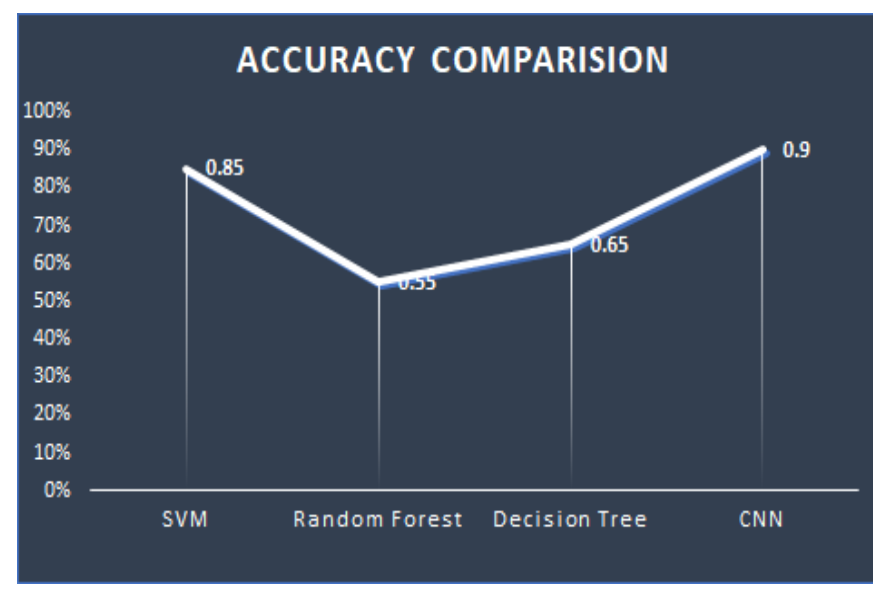

Fig. 1 Accuracy Comparison
According to the value for different models plotted in the image above, it was discovered that CNN had the best among all with an accuracy of $90 \%$. Again, SVM calculation likewise demonstrated an exactness of $85 \%$ which is exceptionally near the precision of CNN. But nearly the accuracy of Random Forest and Decision Tree is not sufficient. They have demonstrated an accuracy of 55\% and $65 \%$ individually.

\section{B. Precision, Recall \& F1-Score}

True positives and true negatives show the perceptions that are accurately predicted and that is why they are marked in red. The false-negative and false-positive have the opposite so they are marked in red. We want to limit false positives and false negatives. The evaluation matrix formula is shown in Fig.2.These terms are somewhat confounding. So how about we take each term individually and comprehend it completely.

\begin{tabular}{|c|c|c|}
\hline & $\begin{array}{c}\mathbf{P}^{\prime} \\
\text { (Predicted) }\end{array}$ & $\begin{array}{c}\mathbf{n}^{\prime} \\
\text { (Predicted) }\end{array}$ \\
\hline $\begin{array}{c}\mathbf{P} \\
\text { (Actual) }\end{array}$ & True Positive & False Negative \\
\hline $\mathbf{n}$ \\
(Actual) & False Positive & True Negative \\
\hline
\end{tabular}

Fig. 2 Evaluation Matrix

Random Forest advantage points include:

- $\quad$ True Positive (TP):

True positives mean proper prediction in the result. Before start modelling the value of the actual class is yes and after the modelling, we predicted the class is yes. E.g., if the actual class predicts the image is belong to the paper class and after prediction, we have found that the class is paper, then we can consider it as true positive.

- $\quad$ True Negative (TN):

True negative also indicates proper prediction but on the negative side. That means the actual value is no and our predicting model also predicts the class as a negative value. Then we consider it True negative. E.g., if the actual class predicts the image is not a metal class and after prediction, we have found that the class is not metal then it is known as true negative.

False-positive and false negatives, these qualities happen when your real class clashes with the anticipated class.

- $\quad$ False Positives (FP):

False-positive occurs when the actual class is no but the model predicts it as a yes. E.g. if the actual class value predicts that the image does not belong to the 
paper class, but the predicted model shows that the image belongs to the paper class.

- $\quad$ False Negative (FN):

False Negative occurs when the actual class is yes, but the model predicts it as a no. E.g. if the actual class value predicts that the image belongs to the paper class but our predicted mode; shows that the image does not belong to that specific class.

\section{Precision}

If we divide the value of actual positives by the sum of true positives and false, positives then we find the precision. The precision comparisons found in our experiment is stated in Fig.3. The Precision scores for different algorithms are mentioned in Table 1 below.

- Precision = actual positives $/$ (true positives + false positives)

TABLE I

PRECISION TABLE

\begin{tabular}{|l|l|l|l|l|l|l|}
\hline & cardboard & glass & metal & paper & plastic & trash \\
\hline SVM & 0.87 & 0.8 & 0.79 & 0.6 & 0.87 & 0.7 \\
\hline $\begin{array}{l}\text { Random } \\
\text { forest }\end{array}$ & 0.62 & 0.44 & 0.45 & 0.64 & 0.58 & 0.61 \\
\hline $\begin{array}{l}\text { Decision } \\
\text { tree }\end{array}$ & 0.63 & 0.65 & 0.54 & 0.81 & 0.6 & 0.58 \\
\hline CNN & 0.98 & 0.8 & 0.86 & 0.9 & 0.91 & 0.63 \\
\hline
\end{tabular}

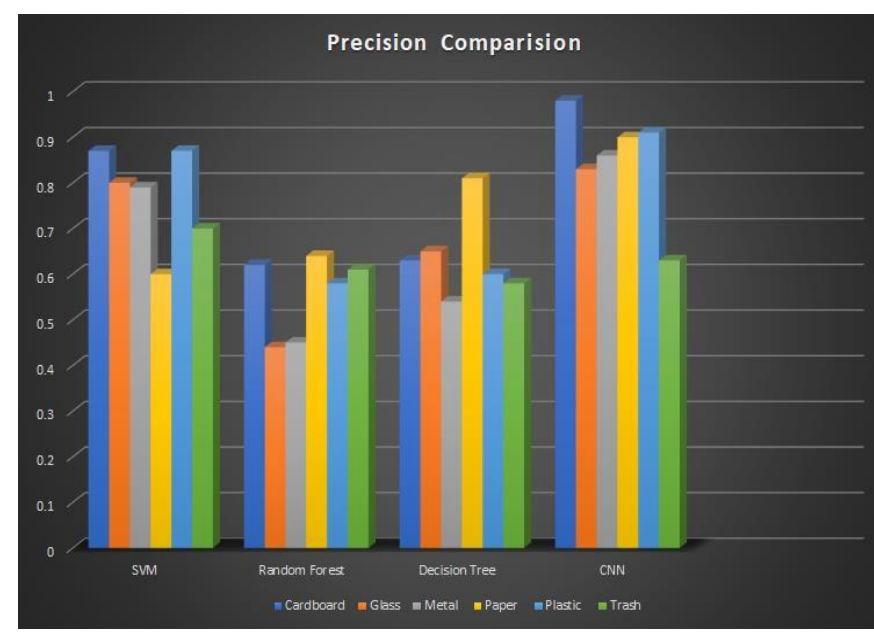

Fig. 3 Precision Comparison

D. Recall

- $\quad$ Recall $=$ True positive $/$ (true positives + false negatives)

The recall comparisons found in our experiment is stated in Fig.4. The Recall scores for different algorithms are mentioned in Table 2 below.
TABLE III

RECALL TABLE

\begin{tabular}{|l|l|l|l|l|l|l|}
\hline & $\begin{array}{l}\text { cardb } \\
\text { oard }\end{array}$ & glass & metal & paper & plastic & trash \\
\hline SVM & 0.81 & 0.8 & 0.85 & 0.6 & 0.82 & 0.83 \\
\hline $\begin{array}{l}\text { Random } \\
\text { forest }\end{array}$ & 0.54 & 0.38 & 0.67 & 0.64 & 0.54 & 0.46 \\
\hline $\begin{array}{l}\text { Decision } \\
\text { tree }\end{array}$ & 0.58 & 0.52 & 0.72 & 0.81 & 0.65 & 0.52 \\
\hline CNN & 0.88 & 0.83 & 0.86 & 0.93 & 0.89 & 0.74 \\
\hline
\end{tabular}

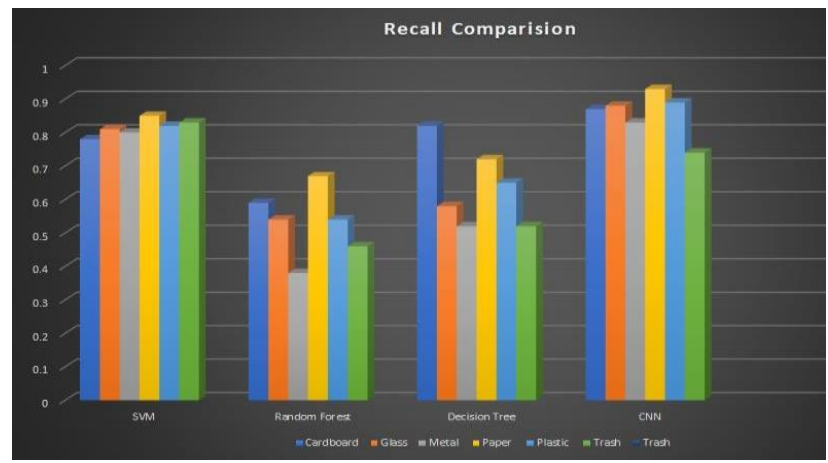

Fig. 4 Recall Comparison

E. F1-Score

F1-Score $=(2 *$ Precision * Recall $) /($ Precision + Recall $)$

The F1-Score comparisons found in our experiment is stated in Fig.5. The scores for different algorithms are mentioned in Table 3 below.

TABLE IIIII

F1-SCORE TABLE

\begin{tabular}{|l|l|l|l|l|l|l|}
\hline & $\begin{array}{l}\text { card } \\
\text { boar } \\
\text { d }\end{array}$ & $\begin{array}{l}\text { glas } \\
\text { s }\end{array}$ & metal & paper & $\begin{array}{l}\text { plast } \\
\text { ic }\end{array}$ & trash \\
\hline SVM & 0.88 & 0.84 & 0.8 & 0.75 & 0.9 & 0.78 \\
\hline $\begin{array}{l}\text { Random } \\
\text { forest }\end{array}$ & 0.61 & 0.48 & 0.41 & 0.66 & 0.56 & 0.52 \\
\hline $\begin{array}{l}\text { Decision } \\
\text { tree }\end{array}$ & 0.71 & 0.61 & 0.53 & 0.76 & 0.62 & 0.55 \\
\hline CNN & 0.92 & 0.86 & 0.84 & 0.91 & 0.9 & 0.68 \\
\hline
\end{tabular}

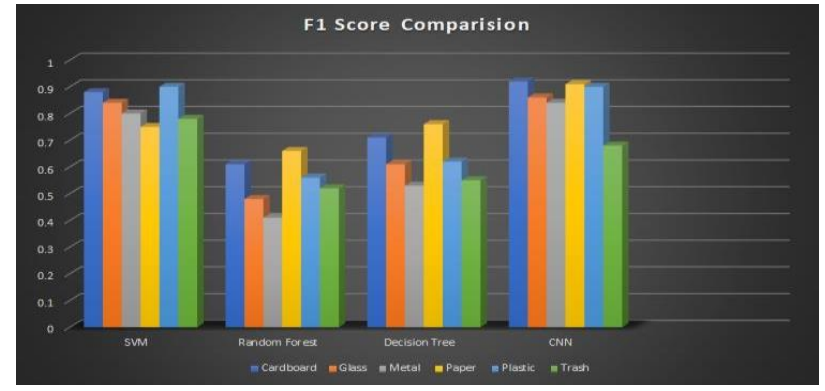

Fig. 5 F1-Score Comparison 


\section{F. Confusion Matrix}

The results of confusion matrixes found in our experiment, using four different algorithms are stated consecutively in Fig.6, Fig.7, Fig.8, and Fig.9.

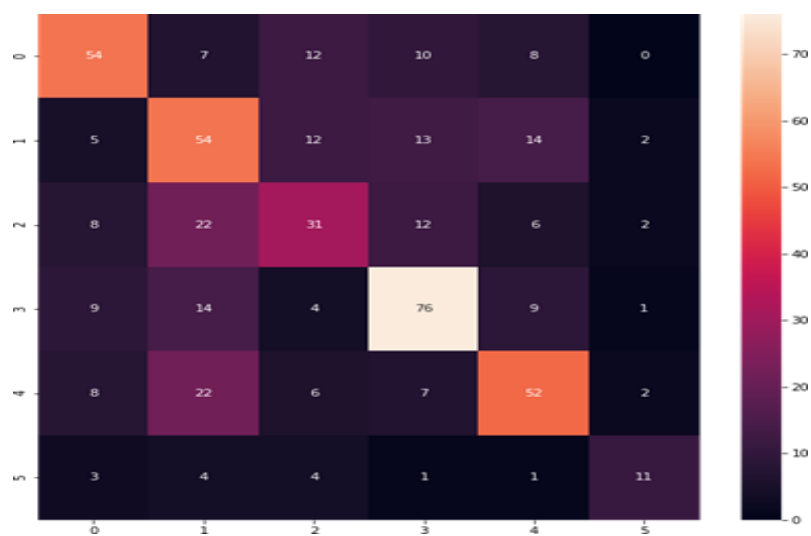

Fig. 6 Confusion Matrix for Random Forest after tuning

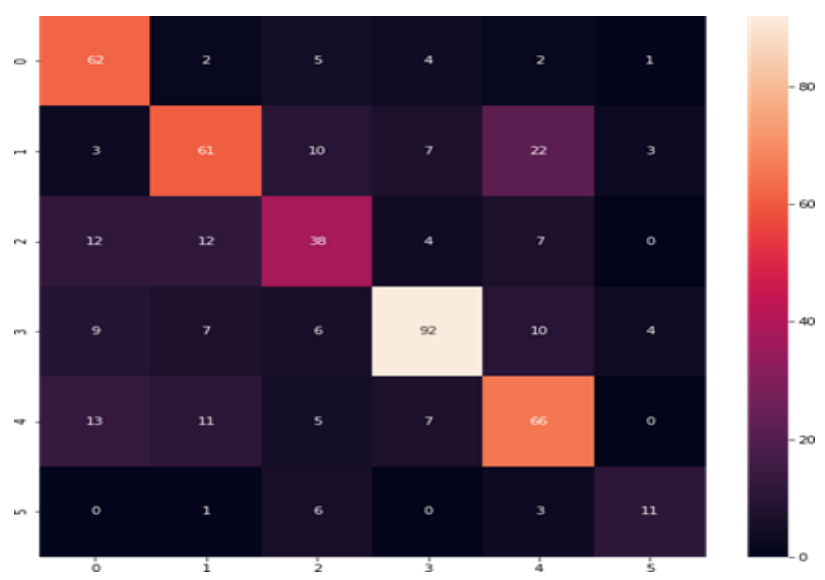

Fig. 7 Confusion Matrix for Decision Tree

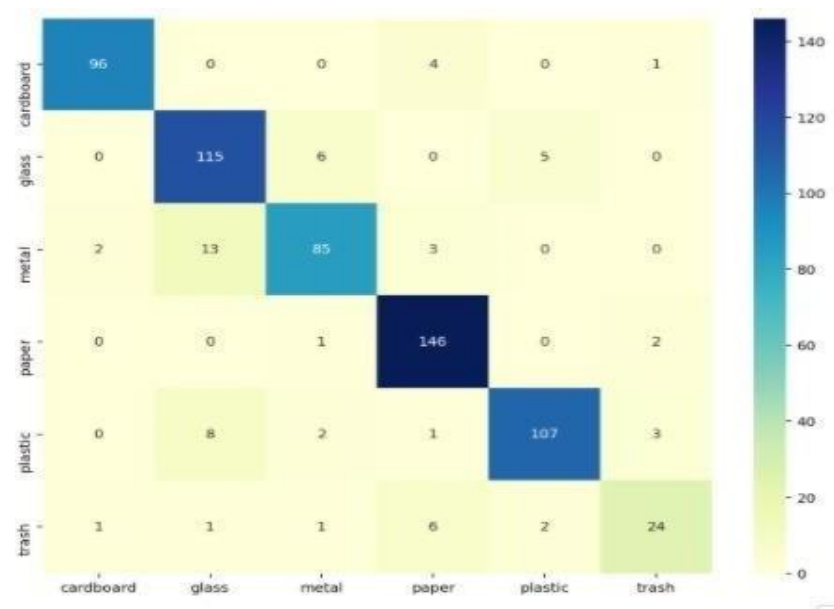

Fig. 8 Confusion Matrix for CNN

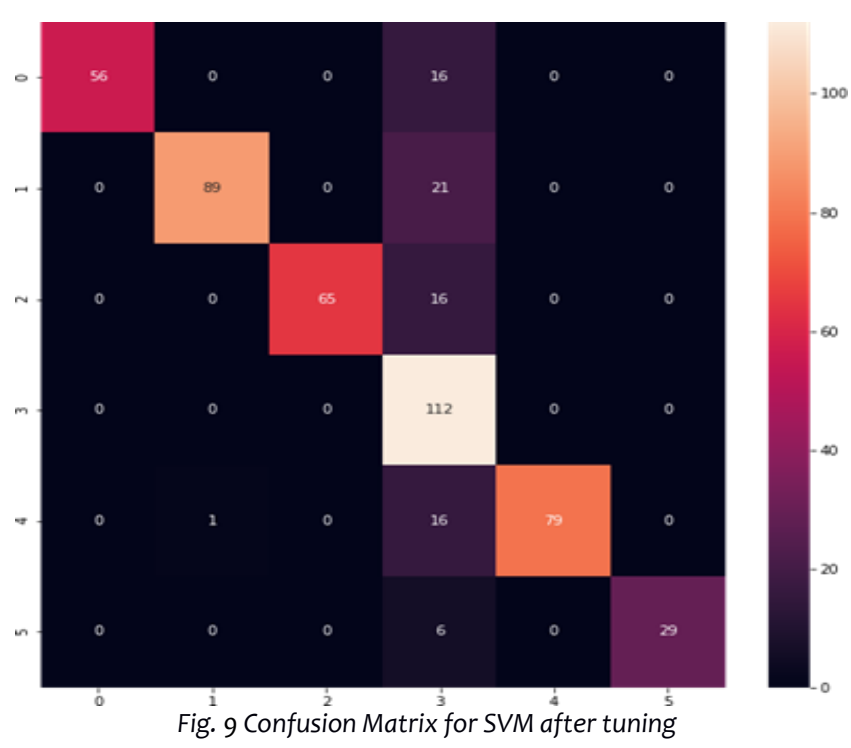

As indicated in the figures depicted above, CNN has shown a lesser amount of waste misclassification among all the experimented algorithms conducted in this research.

\section{CONCLUSIONS}

As indicated by the survey directed and the outcomes assembled, it is very well observed that a CNN system can surpass the presentation of pretty much every model constructed in this research. Boosting any count and receiving it with Cross-Validation plans with various folds, the introduction of any model can be raised. After building the best model for each algorithm using the hyperparameter tuning, $\mathrm{CNN}$ has come up with the best accuracy while SVM is slightly behind. After seeing the accuracy of the Random forest and decision tree, it is clearly shown that they have not performed properly in classifying the waste images properly.

\section{ACKNOWLEDGMENT}

The authors are grateful to Kulliyyah of Information and Communication Technology, International Islamic University Malaysia for their assistance and guidelines.

\section{REFERENCES}

[1] Z. Lenkiewicz, "Waste and the Sustainable Development Goals." Retrieved from Wasteaid: https://wasteaid. org. uk/wastesustainable ..., 2018.

[2] O. Adedeji and Z. Wang, "Intelligent waste classification system using deep learning convolutional neural network," Procedia Manuf., vol. 35, pp. 607-612, 2019.

[3] M. Pamintuan, S. M. Mantiquilla, H. Reyes, and M. J. Samonte, "i-BIN: An Intelligent Trash Bin for Automatic Waste Segregation and Monitoring System," in 2019 IEEE 11th International Conference on Humanoid, Nanotechnology, Information Technology, Communication and Control, Environment, and Management (HNICEM), pp. 1-5.

[4] A. Silva and E. Soares, "Artificial intelligence in automated sorting in trash recycling," XV Encontro Nac. Inteligência Artif. e Comput., 2018.

[5] X. Xu, X. Qi, and X. Diao, "Reach on Waste Classification and 
Identification by Transfer Learning and Lightweight Neural Network," 2020.

[6] Z. Oralhan, B. Oralhan, and Y. Yiğit, "Smart city application: Internet of things (IOT) technologies based smart waste collection using data mining approach and ant colony optimization," Internet Things, vol. 14, no. 4, p. 5, 2017.

[7] P. Cp et al., "ICACCl-02 (A): Artificial Intelligence and Machine Learning/Data Engineering/Biocomputing (Regular Papers)."

[8] G. E. Sakr, M. Mokbel, A. Darwich, M. N. Khneisser, and A. Hadi, "Comparing deep learning and support vector machines for autonomous waste sorting," in 2016 IEEE International Multidisciplinary Conference on Engineering Technology (IMCET), 2016, pp. 207-212.

[9] G. Mittal, K. B. Yagnik, M. Garg, and N. C. Krishnan, "Spotgarbage: smartphone app to detect garbage using deep learning," in Proceedings of the 2016 ACM International Joint Conference on Pervasive and Ubiquitous Computing, 2016, pp. 940-945.

[10] I. F. Hanbal, J. S. Ingosan, N. A. A. Oyam, and Y. Hu, "Classifying Wastes Using Random Forests, Gaussian Naïve Bayes, Support Vector Machine and Multilayer Perceptron," in IOP Conference Series: Materials Science and Engineering, 2020, vol. 803, no. 1, p. 12017.

[11] N. S. Gupta, V. Deepthi, M. Kunnath, P. S. Rejeth, T. S. Badsha, and B. C. Nikhil, "Automatic Waste Segregation," in 2018 Second International Conference on Intelligent Computing and Control Systems (ICICCS), 2018, pp. 1688-1692.
[12] R. S. S. Devi, V. R. Vijaykumar, and M. Muthumeena, "Waste Segregation using Deep Learning Algorithm."

[13] Y. Chu, C. Huang, X. Xie, B. Tan, S. Kamal, and X. Xiong, "Multilayer hybrid deep-learning method for waste classification and recycling," Comput. Intell. Neurosci., vol. 2018, 2018.

[14] Y. Zhao and Y. Zhang, "Comparison of decision tree methods for finding active objects," Adv. Sp. Res., vol. 41, no. 12, pp. 1955-1959, 2008.

[15] A. Bosch, A. Zisserman, and X. Munoz, "Image classification using random forests and ferns," in 2007 IEEE 11th international conference on computer vision, 2007, pp. 1-8.

[16] M. Yang and G. Thung, "Classification of trash for recyclability status," CS229 Proj. Rep., vol. 2016, 2016.

[17] C. Agarwal and A. Sharma, "Image understanding using decision tree based machine learning," in ICIMU 2011: Proceedings of the 5th international Conference on Information Technology \& Multimedia, 2011, pp. $1-8$.

[18] C. Cortes, "WSupport-vector network," Mach. Learn., vol. 20, pp. 1$25,1995$.

[19] E. A. Bender, "Mathematical methods in artificial intelligence," 1996.

[20] K. Millard and M. Richardson, "On the importance of training data sample selection in random forest image classification: A case study in peatland ecosystem mapping," Remote Sens., vol. 7, no. 7, pp. $8489-8515,2015$. 